\title{
BMJ Open Cost-minimisation analysis alongside a pilot study of early Tissue Doppler Evaluation of Diastolic Dysfunction in Emergency Department Non-ST Elevation Acute Coronary Syndromes (TEDDy-NSTEACS)
}

\author{
Vijay S Gc, ${ }^{1,2}$ Mohamad Alshurafa, ${ }^{3}$ David J Sturgess, ${ }^{\circ}$ Joseph Ting, ${ }^{3}$ \\ Kye Gregory, ${ }^{3}$ Ana Sofia Oliveira Gonçalves, ${ }^{2}$ Jennifer A Whitty ${ }^{2}$
}

To cite: Gc VS, Alshurafa M, Sturgess DJ, et al. Costminimisation analysis alongside a pilot study of early Tissue Doppler Evaluation of Diastolic Dysfunction in Emergency Department Non-ST Elevation Acute Coronary Syndromes (TEDDy-NSTEACS). BMJ Open 2019;9:e023920. doi:10.1136/ bmjopen-2018-023920

- Prepublication history for this paper is available online. To view these files, please visit the journal online (http://dx.doi. org/10.1136/bmjopen-2018023920).

Received 29 May 2018 Revised 7 March 2019 Accepted 17 April 2019

Check for updates

(C) Author(s) (or their employer(s)) 2019. Re-use permitted under CC BY-NC. No commercial re-use. See rights and permissions. Published by BMJ.

${ }^{1}$ Centre for Health Economics, University of York, York, UK ${ }^{2}$ Norwich Medical School, University of East Anglia, Norwich, UK

${ }^{3}$ Mater Research Institute, University of Queensland (MRI-UQ), South Brisbane, Queensland, Australia

Correspondence to Professor Jennifer A Whitty; Jennifer.Whitty@uea.ac.uk

\section{ABSTRACT}

Objective To estimate the cost implications of early angiography for patients with suspected non-ST elevation acute coronary syndrome (NSTEACS) using tissue Doppler imaging (TDI).

Design A decision tree model was used to synthesise data from the pilot study and literature sources. Sensitivity analyses tested the impact of assumptions incorporated into the analysis.

Setting Emergency department (ED), Brisbane, Australia. Participants Patients with suspected NSTEACS. Interventions TDI as a diagnostic tool for triaging patients within 4 hours of presentation in addition to conventional risk stratification, compared with conventional risk stratification alone.

Data sources Resource used for diagnosis and management were recorded prospectively and costed for 51 adults who had echocardiography within 24 hours of admission. Costs for conventional care were based on observed data. Cost estimates for the TDI intervention assumed patients classified as high risk at TDI (E/e'>14) progressed early to angiography with an associated 1-day reduction in length of stay.

Primary outcome measures Costs until discharge from the Australian healthcare perspective in 2016-2017 prices.

Results Findings suggest that using TDI as a diagnostic tool for triaging patients with suspected NSTEACS is likely to be cost saving by $\$$ A1090 (95\% credible interval: $\$ A 573$ to $\$ A 1703$ ) per patient compared with conventional care. The results are mainly driven by the assumed reduction in length of stay due to the inclusion of early TDI in clinical decision-making.

Conclusions This pilot study indicates that compared with conventional risk stratification, triaging patients presenting with suspected NSTEACS with TDI within 4 hours of ED presentation has potential cost savings. Findings assume a reduction in hospital stay is achieved for patients considered to be high risk at TDI. Larger, comparative studies with longer follow-up are needed to confirm the clinical effectiveness of TDI as a diagnostic
Strengths and limitations of this study

- The economic evaluation used evidence on healthcare resource use and probabilities prospectively obtained alongside a pragmatic pilot study to assess the prognostic utility of tissue Doppler imaging (TDI) in patients with suspected non-ST elevation acute coronary syndrome.

- The analysis was based on data from a non-comparative pilot study, and assumed a hypothetical (rather than observed) reduction in hospital length of stay would result from early progression to angiography in patients classified as 'high risk' if TDI was performed within 4 hours of hospital admission.

- The decision-analytical model assumes equal clinical outcome regardless of triaging strategy and did not include potential long-term outcomes, for example, survival and quality of life.

strategy for NSTEACS, the assumed reduction in hospital stay and any cost saving.

\section{INTRODUCTION}

Acute coronary syndrome (ACS) is a significant contributor to both morbidity and mortality globally, accounting for almost half of all deaths related to cardiovascular disease. ${ }^{1}$ It comprises a broad spectrum of clinical presentations including ST-elevated ACS and non-ST elevated ACS (NSTEACS). In developed economies, incident rates of ACS are declining but they remain one of the main contributors of premature death in adults. ${ }^{2}$ Suspected ACS represents a substantial financial burden to the healthcare system as $20 \%$ of patients are rehospitalised within 6 months of their initial admission. ${ }^{3}$ In Australia, ACS 
accounts for $5 \%-10 \%$ of all emergency department (ED) presentations ${ }^{4}$ and is responsible for approximately 75000 hospital admissions and 10000 deaths annually. ${ }^{5}$

Over two-thirds $(65 \%-70 \%)$ of all myocardial infarctions are categorised as NSTEACS. ${ }^{6}$ Management of patients with a working diagnosis of NSTEACS depends on risk stratification. ${ }^{7}$ Early identification of low-risk individuals improves effectiveness of care to those in highest need and helps prevent unnecessary admission to hospital. Thus, early diagnosis of NSTEACS is expected to reduce the huge burden of care in terms of morbidity, mortality and costs. ${ }^{8}$ Previous studies in Australia indicate that early identification of an intermediate-risk group and use of an accelerated diagnostic approach such as the Brisbane protocol would be a cost-saving approach as they are associated with lower expected costs and length of stay (LoS) ${ }^{9}{ }^{10}$ Current guidelines recommend the time to angiography to be less than 24 hours for 'high risk' and less than 72 hours for the remainder of patients. ${ }^{11}$ However, the management and risk stratifications in practice remain suboptimal. In one study, only half of the NSTEACS patients underwent guideline-recommended invasive management. ${ }^{12}$

Incorporation of diagnostic tools such as tissue Doppler imaging (TDI) for the non-invasive evaluation of diastolic function in critical settings such as the ED is time efficient, inexpensive and could help improve risk stratification. ${ }^{13}$ Thus, these tools have the potential to improve the management of patients with suspected NSTEACS. The TDI parameter, that is, the ratio of early transmitral flow velocity to early diastolic septal mitral annulus velocity $\left(\mathrm{E} / \mathrm{e}^{\prime}\right)$ is a powerful predictor of cardiac ischaemia and subsequent mortality. ${ }^{14}$ Echocardiography can be safely used to evaluate cardiac diastolic function at the bedside. The patient-important qualities favouring TDI are numerous and include its acceptability to the patient, applicability at bedside, ease of use at point of care, a high level of concordance between observers, ${ }^{15}$ reproducibility, validity, feasibility and prognostic utility. ${ }^{16}$

The purpose of this cost-minimisation analysis was to evaluate the cost implications of TDI-guided (E/e'>14) risk stratification of suspected NSTEACS patients from 'low or intermediate risk' to 'high risk' and early angiography (within 4 hours of presentation at ED) compared with standard (usual) care. The National Emergency Access Target stipulates that a predetermined proportion of patients should be admitted, discharged or transferred from the ED within 4 hours of presentation. ${ }^{17}$ Thus, the 4 hours benchmark is compatible with current guidelines to pursue definitive reperfusion within 8-12hours of onset of ischaemic pain.

\section{METHODS}

A cost-minimisation decision analysis was undertaken from the healthcare perspective, to compare the costs of utilising TDI within 4 hours of ED presentation in addition to conventional risk stratification as a triaging tool for subsequent angiography for adults with suspected NSTEACS, compared with conventional risk stratification alone. Data for the decision-analytical model were primarily obtained from an Australian cohort study (the Tissue Doppler Evaluation of Diastolic Dysfunction in Emergency Department Non-ST Elevation Acute Coronary Syndromes (TEDDy-NSTEACS) study). Our study was undertaken accordingly to the Consolidated Health Economic Evaluation Reporting Standards (CHEERS) guidelines of reporting health economic evaluations. ${ }^{18}$

\section{The TEDDy-NSTEACS study}

The TEDDy-NSTEACS study was a prospective cohort study conducted at the Mater Hospital Brisbane between August 2014 and October 2015. Adults ( $\geq 18$ years) presenting to the ED with chest pain and admitted to the coronary care unit (CCU) for further diagnostic, prognostic and acute management of suspected NSTEACS were approached for enrolment in this observational cohort study. Patients enrolled in the study had an echocardiography within 24 hours of admission in addition to standard of care and an E/e' was calculated. As this was an observational cohort study, comparative clinical outcomes were not investigated for different triaging strategies. However, since the intent of triaging with TDI in addition to conventional risk stratification is to reach a quicker diagnosis, this economic evaluation assumes equivalent clinical outcomes regardless of triaging strategy. We consider this to be a conservative assumption. Therefore, a cost-minimisation approach is applied.

The primary prognostic variables of interest for the related prognostic study were mean $\mathrm{E} / \mathrm{e}^{\prime}$ and serum biopeptide N-Terminus B-type natriuretic peptide (NT-proBNP). We hypothesise that TDI performed at the point of care in ED patients admitted for chest pain and suspected of NSTEACS may reduce expenditure by improving the accuracy of triage and reducing resource utilisation. Use of echocardiography to more accurately predict patients who are at a higher risk of an NSTEACS compared with conventional triaging may be cost-effective. Our data demonstrated NT-proBNP to be inferior to TDI in risk stratification of NSTEACS. It is not considered in this economic evaluation because our clinical data do not support its translation to clinical use for this purpose.

\section{Diagnostic strategies}

The patients presenting at the ED with chest pain in the cohort study were triaged using conventional risk stratification and management using American College of Cardiology and American Heart Foundation.${ }^{19}$ Thus, we first measured the resource utilisation and estimated the cost of standard (usual) care for managing and treating patients with suspected NSTEACS from ED presentation to hospital discharge. The resource utilisation for the observational cohort was used for the comparator arm in the decision-analytical model.

The intervention of interest for the model was the application of TDI within 4 hours of presentation to ED 
as a triaging tool, to guide management. In addition to the conventional risk stratification for patients suspected of NSTEACS, TDI was performed during the admission. The E/e' was measured to evaluate the prognostic utility of TDI in patients with suspected NSTEACS using a threshold of mean E/e'>14 based on the most recent recommendations by the American Society of Echocardiography and European Association of Cardiac Imaging. ${ }^{20}$

Although E/e' was measured in the cohort study, it was not used in clinical decision-making in the study. Thus, we estimated the short-term costs from admission to discharge of a hypothetical diagnostic strategy involving TDI within 4 hours of admission, based on clinical judgement of two authors (MA and DJS) what would have been assumed to occur to patients, should the TDI results have been acted on. In the TDI strategy, patients with E/e'>14 were considered high risk, and were assumed to progress directly to angiogram for diagnosis. Diagnostic parameters (sensitivity and specificity) performed early during initial hospitalisation as compared with the gold-standard angiogram for diagnosing NSTEACS were used. When TDI was used as a diagnostic tool for non-ST elevation myocardial infarction (NSTEMI) compared with angiogram as gold standard, the specificity of E/e' $>14$ to detect NSTEMI was $86 \%$ (95\% CI $0.72 \%$ to $0.95 \%$ ) and sensitivity of $67 \%$ ( $95 \%$ CI $0.30 \%$ to $0.93 \%$ ).

In patients with coronary disease, a review of the literature on diastolic dysfunction concluded that the evaluation of diastolic dysfunction has diagnostic and prognostic roles in the management of ACS. ${ }^{21} 22$ TDI in the form of E/e' has emerged as a superior predictor of survival after first acute myocardial infarction relative to other clinical or echocardiographic features. ${ }^{1423}$ TDI has proven incremental prognostic value with respect to routine clinical, laboratory and imaging information. ${ }^{24}{ }^{25}$ The predictive power of E/e' has been recently confirmed in patients that underwent coronary angiography after their first ever NSTEMI. ${ }^{26}$
The analysis was conducted in two parts. The first analysis involved costing of resource utilisation for the cohort of 51 patients included in the pilot study to indicate the costs of conventional triaging. Subsequently, we performed a cost-minimisation analysis to compare the costs of the hypothetical TDI triaging intervention to that of conventional triaging.

\section{Resource use}

Data describing in-hospital resource utilisation related to the diagnosis and management of ACS episodes were captured with urgency-related group (URG) and diagnostic-related group (DRG) codes, and electronic medical chart review. Resources such as diagnostic tests (echocardiography scan, echocardiogram, dobutamine stress echocardiogram, stress test and coronary angiography), staff time (echocardiographer and echocardiologist), hospital transfers, high care and LoS from admission to discharge were extracted from the discharge summaries and pathology databases. Costings for the ED and inpatient episodes were based on the assigned URG and DRG price weights, ${ }^{27}$ respectively. The cost of ED visit was calculated by multiplying the base payment (national efficient price) by the relative weight of each URG while the cost of an inpatient episode was calculated using the base payment, the relative weight of each DRG, LoS and an intensive care unit adjustment, reflecting the increased cost of time spent in the CCU). Costs of diagnostic tests were sourced from the Medicare Benefits Schedule. ${ }^{28}$ Thus, the total cost per patient refers to an average cost of hospital stay, procedures and resource use from admission to discharge. Resources used and their unit costs in Australian dollars at 2016 values along with sources are presented in table 1 .

\section{Model overview}

A decision-analytical model was developed in Microsoft Excel, consistent with the standard treatment pathways

\begin{tabular}{lll}
\hline Table 1 Unit costs & & \\
\hline Cost item & Unit cost $\mathbf{( \$ A )}$ & Source \\
\hline CT pulmonary angiography & 510.00 & MBS 2016, ${ }^{28}$ code 57350 \\
Coronary CT angiography & 700.00 & MBS 2016, ${ }^{28}$ code 57360 \\
\hline Dobutamine stress echocardiogram & 261.65 & MBS $2016,{ }^{28}$ code 55117 \\
Echocardiography with E/e' measurement & 53.79 & $\begin{array}{l}\text { Echocardiographer's hourly rate (employee } \\
\text { payscale) })^{33}\end{array}$ \\
\hline Echocardiography without E/e' measurement & 34.06 & Mater Hospital data, assumed 45 min \\
ECG & 31.25 & MBS 2016, ${ }^{28}$ code 11700 \\
\hline Exercise stress test echography & 261.65 & MBS 2016, ${ }^{28}$ code 55116 \\
\hline Exercise stress test ECG & 152.15 & MBS 2016, ${ }^{28}$ code 11712 \\
\hline Hospital transfer & 671.00 & Queensland Health \\
\hline Invasive angiogram & 354.90 & MBS 2016, ${ }^{28}$ code 38215 \\
\hline
\end{tabular}

MBS, Medicare Benefits Schedule. 


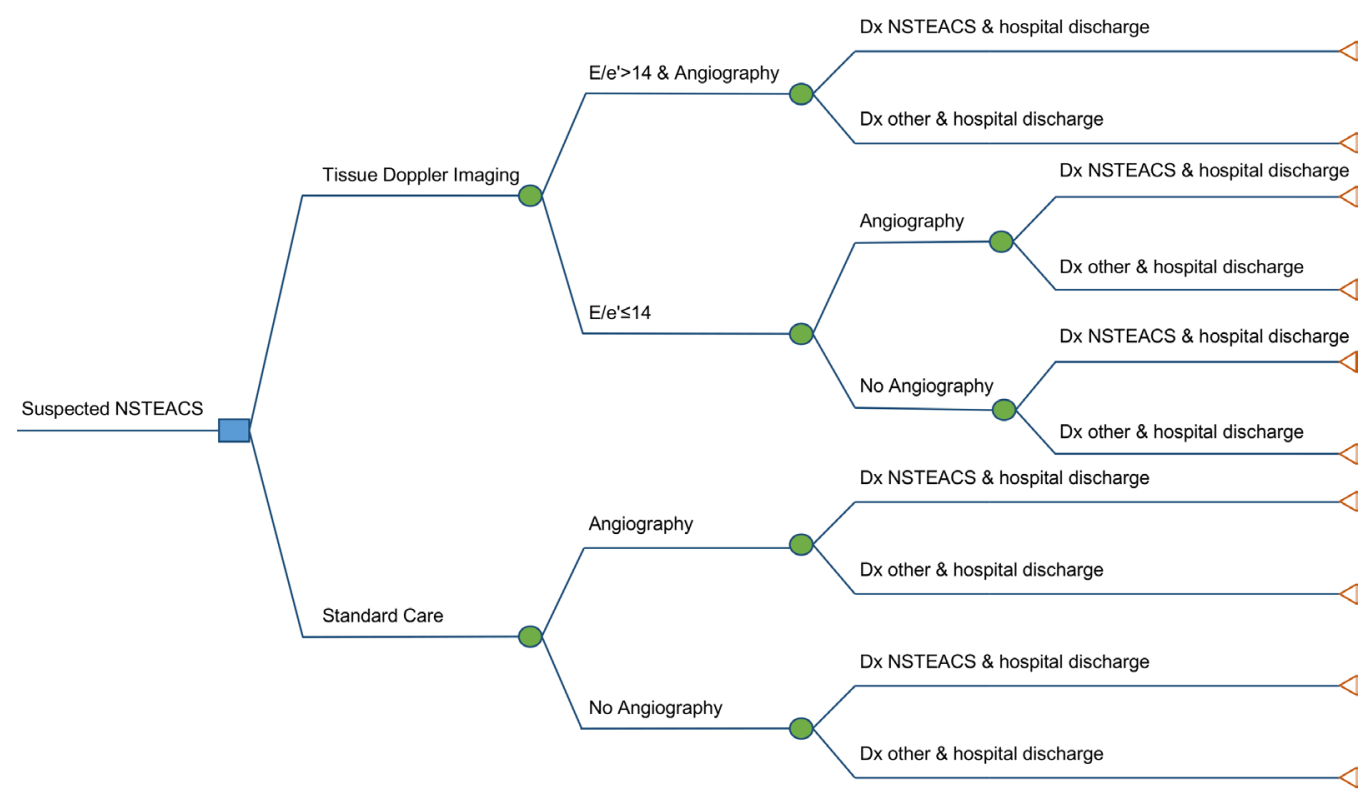

Figure 1 Decision tree for suspected NSTEACS patients. Dx NSTEACS, diagnosis of NSTEACS; Dx other, diagnosis of other than NSTEACS; NSTEACS: non-ST elevation acute coronary syndrome.

for patients with suspected NSTEACS, to compare the costs of proceeding to early angiography for patients with E/e'>14, compared with standard care. The decision tree (figure 1) consisted of TDI and standard care arms leading up to hospital discharge with a diagnosis of NSTEACS or 'other than NSTEACS', assuming equal long-term outcomes. For the TDI (intervention) arm, it was assumed that E/e' was measured and used in clinical decision-making. The cut-off value for the length of time from admission to receiving an echocardiography/ angiography was set to 4 hours. Previous studies evaluating the early implementation of angiography in the ED for the evaluation of ACS indicated a reduction in LoS up to 1 day. ${ }^{29}{ }^{30}$ In this study, we assumed patients with E/e'>14 were high risk and proceeded to angiography within 4 hours of admission, received early treatment and therefore earlier hospital discharge (by 1 day) than the standard care arm (table 2). It was assumed that highrisk patients who had angiography within 4 hours of angiography but had a diagnosis other than NSTEACS would have the same benefit in LoS saving of 1 day, which is associated with quicker diagnosis. However, in the TEDDy-NSTEACS study patients had echocardiography within 24 hours of admission. Patients with E/e' $\leq 14$ were assumed to receive angiography with the probability and timings observed within the pilot study.

For the standard care arm, it was assumed that E/e' was not measured, and that all patients proceed to angiography with the probability and timings observed within the pilot study (table 2). All costs and time duration from admission to receiving echocardiography and LoS from admission to discharge were derived from the cohort of 51 patients. Decision tree probabilities were derived by analysing data from the cohort of 51 patients. These are the mean values. Likewise, unit costs of echocardiography, angiography and ED admission were mean costs. The cost of hospital stay (inpatient) was estimated using weighted average of price weights of 15 DRG codes observed in the cohort study and the costing used the Australian National Weighted Activity Units and AR-DRG prices (2016-17). ${ }^{27}$ The average cost per patient was estimated by multiplying the proportion of patients in different branches of the diagram (figure 1) with the unit costs of the different diagnostic tests, LoS, and hospitalisation. No discounting was applied to costs due to the short time horizon (from hospital admission to discharge).

\section{Sensitivity analysis}

Probabilistic sensitivity analysis using 1000 Monte Carlo simulations was used to account for parameter uncertainty in model inputs. One-way deterministic sensitivity analysis was performed to assess the influence of various parameters on the model results where input parameters $(95 \%$ lower and upper values) were varied one at a time and the remaining values were held at their baseline value. In the base-case analysis, it was assumed that patients who proceeded to TDI within 4 hours of admission had discharge 1 day earlier compared with the standard care $\mathrm{arm}$. We also tested other plausible values (none, half and 2 days reduction in $\mathrm{LoS}$ ) in the scenario analyses.

\section{Patient public involvement}

No patients were involved in the development of the research question or the outcome measures nor the design of the study. There are no plans to disseminate the results of the research to study participants.

\section{RESULTS}

Descriptive characteristics of the participants are listed in table 3. During the study period, 2491 patients with chest pain presented to the Mater Hospital ED. Of those, 
Table 2 Parameters used to estimate diagnosis and treatment pathways

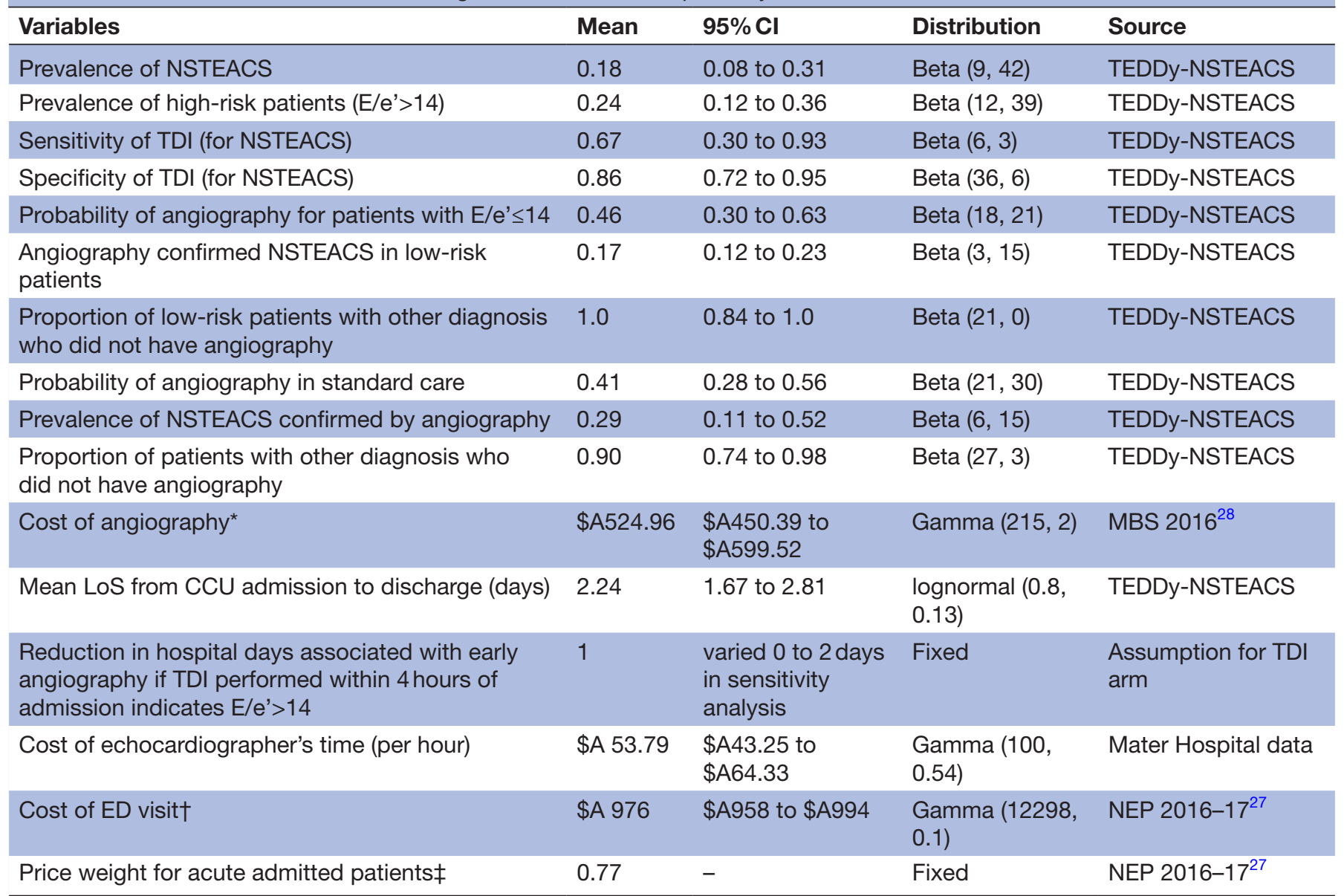

LoS and cost data for standard care arm were based on audit data of 51 patient.

*Weighted average of CTCA $(n=9)$, CTPA $(n=3)$ and invasive angiography $(n=9)$.

tWeighted average of price weights of 5 URG V.1.4 codes: 16, 24, 27, 29 and 86.

fWeighted average of price weights of 15 DRG V.8.0 codes: E61B, F10A, F10B, F14B, F15B, F41A, F41B, F42B, F60A, F69B, F72B, F74A, F74B, F76A and G67B.

CCU, coronary care unit; CTCA, CT coronary angiography; CTPA, CT pulmonary angiography; DRG, diagnostic-related group; ED, emergency department; LoS, length of stay; NEP, National Efficient Price Determination; NSTEACS; non-ST elevation acute coronary syndrome; TDI, tissue Doppler imaging; TEDDy-NSTEACS; Tissue Doppler Evaluation of Diastolic Dysfunction in Emergency Department Non-ST Elevation Acute Coronary Syndromes; URG, urgency-related group.

260 were admitted to the CCU for further evaluation of potential ACS. There were 167 participants suspected of NSTEACS admitted to CCU, of which 51 met inclusion criteria and enrolled in the study.

Table 3 details the average resource use and costs per patient in the pilot study by $\mathrm{E} / \mathrm{e}$ ' ratio. The mean age in this observational cohort of 51 adults was 61 years (range 37-87 years). The mean LoS from ED admission to discharge from the hospital $(\mathrm{n}=51)$ was 2.33 (95\% CI 1.76 to 2.90) days, and patients with $\mathrm{E} / \mathrm{e}^{\prime}>14$ had a mean of 1.04 days (95\% CI -0.29 to 2.36 ) more LoS as compared with those with $\mathrm{E} / \mathrm{e}^{\prime} \leq 14$ (ie, without the TDI result being acted on). The overall mean cost for the pilot study amounted to \$A 15573 per patient. As expected given the 1 day longer average LoS, the mean total cost for the high risk patients $\left(\mathrm{E} / \mathrm{e}^{\prime}>14\right)$ was higher $(\$ \mathrm{~A} 18$ 038) than for low risk $\left(\mathrm{E} / \mathrm{e}^{\prime} \leq 14\right)$ patients $(\$ \mathrm{~A} 14814)$. The preliminary outcome data does suggest that E/e'>14 is concerning for adverse outcome and $\mathrm{E} / \mathrm{e}^{\prime} \leq 14$ reassuring for improved outcome.

The comparative total costs and associated uncertainty from the base-case and sensitivity analyses comparing TDI and standard care are presented in table 4 . In the basecase analysis which assumes acting on TDI is associated with a 1 day reduction in $\mathrm{LoS}$ in those with $\mathrm{E} / \mathrm{e}^{\prime}>14$, the TDI triaging strategy was expected to cost $\$$ A1090 $(95 \%$ credible interval (CrI): \$A573 to \$A1703) less per patient than standard care. Reducing the assumed LoS by a further day (to assume a 2 day reduction in LoS in those with $\left.\mathrm{E} / \mathrm{e}^{\prime}>14\right)$, increases the cost savings estimated for the TDI strategy to \$A 2293 (95\% CrI: \$A 1252 to \$A 3553).

Threshold analysis comparing the cost difference between 0 and 2 days reduction in LoS suggests (figure 2) that the results are robust to uncertainty in all model parameters. As expected, when no reduction in hospital days is assumed, the TDI strategy was not cost saving, but 
Table 3 Patient characteristics and summary of resource use and costs by E/e' in TEDDy-NSTEACS cohort study

\begin{tabular}{|c|c|c|c|}
\hline Variables & $E / e^{\prime} \leq 14(n=39)$ mean (SD) & $E / e^{\prime}>14$ (n=12)mean (SD) & Total $(n=51)$ mean $(S D)$ \\
\hline \multicolumn{4}{|l|}{ Patient characteristics } \\
\hline Age in years, mean (range) & 57 (37-82) & $75(63-87)$ & $61(37-87)$ \\
\hline Male, $\mathrm{n}(\%)$ & $19(49)$ & $1(8)$ & $20(39)$ \\
\hline Hypertension, n (\%) & $30(77)$ & $11(92)$ & $41(80)$ \\
\hline Hyperlipidaemia, n (\%) & $25(64)$ & $11(92)$ & $36(71)$ \\
\hline Diabetes, n (\%) & $7(18)$ & $5(42)$ & $12(24)$ \\
\hline Prior CABG, $n(\%)$ & $1(3)$ & $4(33)$ & $5(10)$ \\
\hline CKD, n (\%) & $3(8)$ & $3(25)$ & $6(12)$ \\
\hline Prior MI, n (\%) & $3(8)$ & $2(17)$ & $5(10)$ \\
\hline TIMI Score>2, n (\%) & $12(31)$ & $10(83)$ & $22(43)$ \\
\hline NT-proBNP, mean (range) & $387(6-6045)$ & 2027 (112-8098) & $787(6-8098)$ \\
\hline E/e', mean (range) & $9.09(5.4-13.1)$ & $19.4(14.6-24)$ & $11.5(5.9-24)$ \\
\hline$E^{\prime}$, mean (range) & $7.7(4.5-10.4)$ & $5.1(2.5-7.4)$ & $7.1(2.5-10.4)$ \\
\hline \multicolumn{4}{|l|}{ Resource use } \\
\hline LoS in ED (in hours) & $1.99(2.78)$ & $2.62(4.12)$ & $2.13(3.11)$ \\
\hline LoS in CCU (in days) & $2.00(1.75)$ & $3.01(2.69)$ & $2.24(2.02)$ \\
\hline Hospital days & $2.08(1.77)$ & $3.12(2.64)$ & $2.33(2.03)$ \\
\hline $\begin{array}{l}\text { Time from ED admission to } \\
\text { echocardiography (in hours) }\end{array}$ & $14.69(7.15)$ & $15.01(9.05)$ & $14.76(7.54)$ \\
\hline \multicolumn{4}{|l|}{ Costs $(\$ A)$} \\
\hline Cost of ED visit & $984(61)$ & $950(64)$ & $976(63)$ \\
\hline CCU cost & 13177 (8545) & $16391(10587)$ & 13934 (9 058) \\
\hline Cost of echocardiography scan & $34.06(12.48)$ & $31.49(9.16)$ & 33.45 (11.75) \\
\hline Total cost per patient & 14814 (8825) & 18038 (10 890) & 15573 (9 338) \\
\hline
\end{tabular}

E/e': ratio of early transmitral flow velocity to spectral tissue peak early diastolic velocity at mitral annulus. e': spectral tissue peak early diastolic velocity at mitral annulus.

CABG, coronary artery bypass graft; CCU, coronary care unit; CKD, chronic kidney disease; ED, emergency department; ; MI, myocardial infarction; NT-proBNP, N-Terminal B-type natriuretic peptide; TEDDy-NSTEACS, Tissue Doppler Evaluation of Diastolic Dysfunction in Emergency Department Non-ST Elevation Acute Coronary Syndromes; TIMI, thrombolysis in myocardial infarction.

rather was estimated to cost $\$$ A113 (95\% CrI: $\$$ A10 to \$A214) per patient more than standard care.

The one-way deterministic sensitivity analyses (figure 3) indicate that the base-case point estimate is most sensitive to changes in prevalence of the high-risk group $\left(\mathrm{E} / \mathrm{e}^{\prime}>14\right)$, the probability of other diagnosis in low-risk group $\left(\mathrm{E} / \mathrm{e}^{\prime} \leq 14\right)$ who did not have angiography, prevalence of angiography in the cohort, and costs associated with angiography and echocardiography. The base-case cost saving was not very sensitive to the sensitivity and specificity of TDI.

\section{DISCUSSION}

\section{Summary of findings}

Our findings provide support for the hypothesis that triaging patients presenting with suspected NSTEACS to have an angiography based on TDI within 4 hours of presentation at the ED in addition to conventional risk stratification is likely to be a cost-saving intervention. This is promising but the large CI around the point estimate highlights uncertainty in the results and the small sample size in the cohort study whose data underpin the model. Moreover, as the TEDDy-NSTEACS was a pilot study without an observed comparator, the data underpinning the model were based on assumptions around key variables (in particular, LoS). Nevertheless, the TDI strategy was cost saving under plausible assumptions tested in the model. Originally, an economic evaluation was proposed alongside this cohort study with the expectation that the proposed TDI intervention would rule out patients who tested negative for NSTEACS according to the angiogram, thus allowing their early discharge. However, the clinical study showed that this diagnostic tool is particularly robust in detecting major adverse cardiac event (MACE) but less robust in detecting negative predictive values.

This is consistent with prior studies that have indicated the threshold of 14 for $\mathrm{E} / \mathrm{e}$ ' is highly specific 
Table 4 Base-case and sensitivity analyses

\section{Cost per patient (\$A)}

\begin{tabular}{|c|c|c|c|}
\hline & \\
\hline & TDI & Standard care & Incremental cost \\
\hline $\begin{array}{l}\text { Base-case analysis (LoS } \\
\text { reduced by } 1 \text { day*) }\end{array}$ & $\begin{array}{l}15338 \\
(12665 \text { to } 18409)\end{array}$ & $\begin{array}{l}16431 \\
(13823 \text { to } 19459)\end{array}$ & $\begin{array}{l}-1090 \\
(-1703 \text { to }-573)\end{array}$ \\
\hline No reduction in LoS† & $\begin{array}{l}16538 \\
\text { (13984 to } 19602)\end{array}$ & $\begin{array}{l}16425 \\
(13877 \text { to } 19505)\end{array}$ & $\begin{array}{l}113 \\
(10 \text { to } 214)\end{array}$ \\
\hline LoS reduced by 2 days* & $\begin{array}{l}14165 \\
\text { (11272 to } 17439)\end{array}$ & $\begin{array}{l}16458 \\
(13838 \text { to } 19580)\end{array}$ & $\begin{array}{l}-2293 \\
(-3553 \text { to }-1252)\end{array}$ \\
\hline
\end{tabular}

Values are mean and $95 \%$ credible interval.

${ }^{*}$ Reduction in hospital LoS from progression to early angiography, which is assumed to occur for those in whom TDI within 4 hours of admission indicates E/e'>14.

†The mean costs in the table for TDI and standard care, when no reduction in LoS is assumed, are close to but not exactly equal due to the bootstrapping method and rounding error.

LoS, length of stay; TDI, tissue Doppler imaging.

for measuring left ventricular diastolic dysfunction (LVDD).${ }^{20}$ Although angiography within 4 hours is desirable, it requires high-end equipment, sufficient expertise in data acquisition and image interpretation, and appropriate patient selection which limits broad implementation of cardiac CT in the emergency room. In order to stratify patients in the cohort, low or high risk, E/e'values were used. However, the decision to proceed to angiography is also based on ongoing chest pain, rising troponin I (TnI), heart failure, and possibly excludes patients with poor premorbid level of function.

Conventional management uses biomarkers of ischaemia (troponins), ECG and CT coronary angiography, to diagnose NSTEACS. ${ }^{19}$ Carefully weighing the costs and benefits of the addition of TDI (E/e') to the conventional

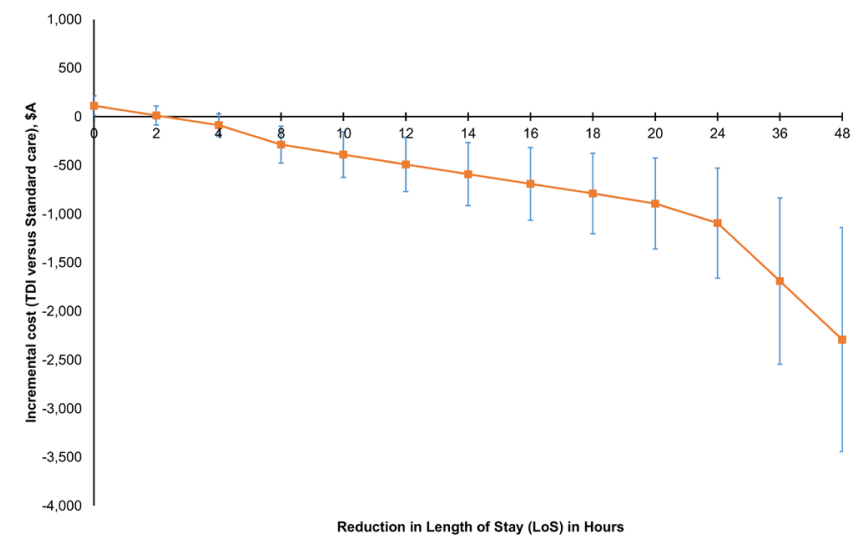

Figure 2 Probabilistic sensitivity analysis: variation of the reduction in length of stay (LoS) with TDI (range 0-48 hours) and the impact on the incremental cost (TDI vs standard care). Point estimates are mean costs in 2016-2017 Australian dollars. Vertical lines refer to variability in point estimates, that is, 95\% credible intervals around incremental cost (\$A). TDI, tissue Doppler imaging. management may prove it to be an optimal strategy for the management of patients presenting with suspected NSTEACS. An improvement in hospital costs can be achieved by early accurate diagnosis and risk stratification. ${ }^{22}$ In previous studies, patients who tested positive for E/e'>14 are at an elevated risk for adverse outcomes and thus may benefit from receiving immediate medical management or coronary intervention. ${ }^{21}$ If TDI improves patient risk stratification and prognostication and reduces LoS, it will likely be associated with decreased healthcare costs. Data are scant regarding the feasibility of using left ventricular diastolic function as a cost-saving risk stratification tool for adults admitted to hospital with suspected NSTEACS. In patients with acute chest pain suspected of NSTEACS, E/e'>14 may provide additional healthcare

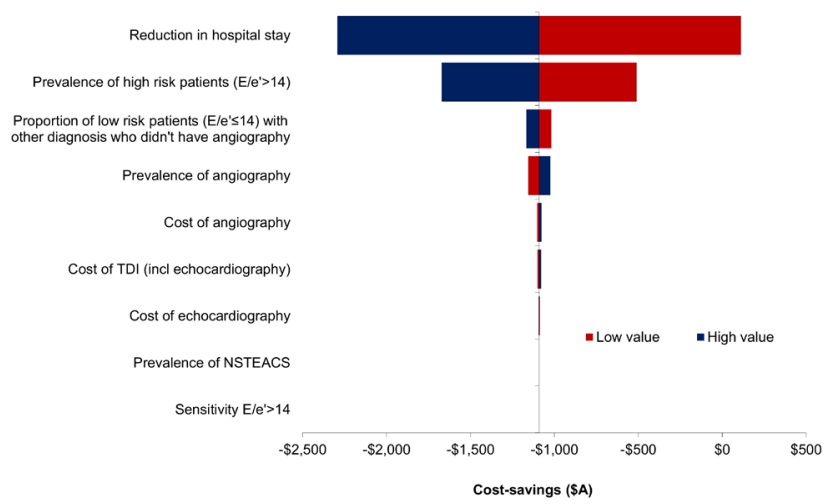

Figure 3 Tornado diagram showing a series of one-way sensitivity analyses comparing standard care with TDI. The vertical line represents the base-case cost savings (\$A1090) and the tails of each bar indicate the changes in cost savings when individual parameter values are varied. NSTEACS, nonST elevation acute coronary syndrome; TDI, tissue Doppler imaging. 
cost saving over and above current clinical, biochemical parameters, risk scores, that are regularly performed. This pilot study supports early measurement of LVDD in acute chest pain management.

\section{Strengths and limitations}

This analysis is indicative only. It is based on a single cohort pilot study with relatively small sample size $(n=51)$ with no direct comparative data; consequently, the economic analysis is based on a relatively simple decision model. The intervention is hypothetical and the model assumes a 1-day reduction in $\mathrm{LoS}$ for patients with E/e'>14 due to efficiencies with triaging assumed to result from TDI. ${ }^{29} 30$ Direct costs related to the intervention and standard care were collected but could not be complemented by microcosting data. Assumptions made for the intervention arm (eg, in reduction of hospital stay days) were arbitrary. If the prevalences of E/e'>14 or LoS reductions associated with the intervention were higher than assumed in the model, then the model would tend to underestimate any cost savings associated with the intervention, and vice versa. A larger, comparative study would strengthen the evidence of TDI cost-effectiveness, as well as confirming the clinical place of TDI in triaging patients with suspected NSTEACS.

Our model did not include potential long-term outcomes, for example, survival and quality of life; instead, it assumes equal clinical outcome regardless of triaging strategy. Evidence to support this assumption stems from prior research which has demonstrated the strong predictive power of E/e' for myocardial infarction, MACE and all-cause mortality. ${ }^{21-23} 253132$ However, our model results are dominated by the short-term costs of diagnostic tests and treatment rather than by long-term outcomes. It is possible that long-term consequences of implementing TDI within 4 hours of presentation at ED in patients with suspected NSTEACS exist. Thus, the findings of this economic evaluation suggest TDI triaging in the ED is a promising strategy that warrants further investigation to confirm associated clinical and economic outcomes.

\section{CONCLUSIONS}

Based on data from a pilot cohort study, this economic analysis indicates that the addition of TDI to the conventional risk stratification approach for triaging adults with suspected NSTEACS in the ED has the potential to save healthcare costs of approximately $\$ A 1100$ per patient between hospital admission to discharge. However, this study makes a number of assumptions and should be considered to be indicative only. Further studies are needed to confirm the clinical effectiveness of TDI in risk stratification for ACS, reduction in hospital stay and longterm outcomes, and the resulting cost-effectiveness.

Acknowledgements The authors thank Sandhir Prasad, John Morgan, Karam Kostner, Jeffrey Presneill, Przemek Palka, Kathy Davies, Peter Cramp and all members of the nursing staff at the emergency department and coronary care unit at Mater Hospital Brisbane for the recruitment of patients, and most of all, the patients who participated in the study.

Contributors DJS, JT and JAW conceived the study and participated in the design of the study. MA and KG undertook the clinical data acquisition. ASOG and VSG undertook the acquisition and analysis of the health economic data. VSG developed the model, ran the analyses, interpreted the results and wrote the first draft. All authors contributed to analysing and interpreting the data. All authors read, contributed to and approved the final manuscript.

Funding This work was supported by a grant from the Emergency Medicine Foundation (Australia) Queensland Programme.

Competing interests None declared.

Patient consent for publication Obtained.

Ethics approval The study was approved by local institutional human research ethics and governance committees (HREC/13/MHS/85).

Provenance and peer review Not commissioned; externally peer reviewed.

Data sharing statement This economic evaluation manuscript uses effectiveness data from the pilot study which is unpublished but is available from the authors on request. Unit costs for the resource use are taken from previously published studies and or publicly available sources.

Open access This is an open access article distributed in accordance with the Creative Commons Attribution Non Commercial (CC BY-NC 4.0) license, which permits others to distribute, remix, adapt, build upon this work non-commercially, and license their derivative works on different terms, provided the original work is properly cited, appropriate credit is given, any changes made indicated, and the use is non-commercial. See: http://creativecommons.org/licenses/by-nc/4.0/.

\section{REFERENCES}

1. Turpie AG. Burden of disease: medical and economic impact of acute coronary syndromes. Am J Manag Care 2006;12:S430-4.

2. Timmis A. Acute coronary syndromes. BMJ 2015;351:h5153.

3. Goldberg RJ, Currie K, White K, et al. Six-month outcomes in a multinational registry of patients hospitalized with an acute coronary syndrome (the Global Registry of Acute Coronary Events [GRACE]). Am J Cardiol 2004;93:288-93.

4. Chew DP, Scott IA, Cullen L, et al. National Heart Foundation of Australia \& Cardiac Society of Australia and New Zealand: Australian Clinical Guidelines for the Management of Acute Coronary Syndromes 2016. Heart Lung Circ 2016;25:895-951.

5. Nadel J, Hewitt T, Horton D. Acute coronary syndrome in Australia: Where are we now and where are we going? Australas Med J 2014;7:149-56.

6. Australian Commission on Safety and Quality in Health Care. Acute coronary syndromes - the case for improvement. Sydney: ACSQHC, 2015.

7. Acute Coronary Syndrome Guidelines Working Group. Guidelines for the management of acute coronary syndromes 2006. Med J Aust 2006;184:S9-29.

8. Hamm CW, Bassand JP, Agewall S, et al. ESC Guidelines for the management of acute coronary syndromes in patients presenting without persistent ST-segment elevation: The Task Force for the management of acute coronary syndromes (ACS) in patients presenting without persistent ST-segment elevation of the European Society of Cardiology (ESC). Eur Heart J 2011;32:2999-3054.

9. Cullen L, Greenslade J, Merollini K, et al. Cost and outcomes of assessing patients with chest pain in an Australian emergency department. Med J Aust 2015;202:427-32.

10. Cheng Q, Greenslade JH, Parsonage WA, et al. Change to costs and lengths of stay in the emergency department and the Brisbane protocol: an observational study. BMJ Open 2016;6:e009746.

11. Ellis $\mathrm{C}$, Hammett $\mathrm{C}$, French $\mathrm{J}$, et al. A comparison of invasive angiography, revascularisation and time delays delivered to Australian and New Zealand non-ST-elevation myocardial infarction/ unstable angina pectoris (NSTEMI/UAP) patients: results of the 2012 SNAPSHOT Bi-National acute coronary Syndrome (ACS) audit. Heart, Lung and Circulation 2014;23:e23.

12. Chew DP, Amerena J, Coverdale S, et al. Current management of acute coronary syndromes in Australia: observations from the acute coronary syndromes prospective audit. Intern Med J 2007;37:741-8.

13. Greaves SC. Role of echocardiography in acute coronary syndromes. Heart 2002;88:419-25.

14. Hillis GS, Møller JE, Pellikka PA, et al. Noninvasive estimation of left ventricular filling pressure by $\mathrm{E} / \mathrm{e}^{\prime}$ is a powerful predictor of survival after acute myocardial infarction. J Am Coll Cardiol 2004;43:360-7. 
15. Sanderson JE, Wang M, Yu CM. Tissue Doppler imaging for predicting outcome in patients with cardiovascular disease. Curr Opin Cardiol 2004;19:458-63.

16. Sturgess DJ. Transthoracic echocardiography: an overview. In: Lumb P, Karakitsos D, eds. Critical Care Ultrasound: Elsevier Inc, 2015.

17. Sullivan C, Staib A, Khanna S, et al. The National Emergency Access Target (NEAT) and the 4-hour rule: time to review the target. Med $J$ Aust 2016;204:354.

18. Husereau D, Drummond M, Petrou S, et al. Consolidated Health Economic Evaluation Reporting Standards (CHEERS)--explanation and elaboration: a report of the ISPOR Health Economic Evaluation Publication Guidelines Good Reporting Practices Task Force. Value Health 2013;16:231-50.

19. Amsterdam EA, Wenger NK, Brindis RG, et al. 2014 AHA/ACC Guideline for the Management of Patients with Non-ST-Elevation Acute Coronary Syndromes: a report of the American College of Cardiology/American Heart Association Task Force on Practice Guidelines. J Am Coll Cardiol 2014;64:e139-228.

20. Nagueh SF, Smiseth OA, Appleton CP, et al. Recommendations for the evaluation of left ventricular diastolic function by Echocardiography: an update from the American Society of Echocardiography and the European Association of Cardiovascular Imaging. J Am Soc Echocardiogr 2016;29:277-314.

21. Ohara T, Little WC. Evolving focus on diastolic dysfunction in patients with coronary artery disease. Curr Opin Cardiol 2010;25:613-21.

22. Goodacre S, Thokala P, Carroll C, et al. Systematic review, metaanalysis and economic modelling of diagnostic strategies for suspected acute coronary syndrome. Health Technol Assess 2013:17:1-188.

23. Prasad SB, See V, Tan T, et al. Serial Doppler echocardiographic assessment of diastolic dysfunction during acute myocardial infarction. Echocardiography 2012;29:1164-71.

24. Richardson-Lobbedez M, Maréchaux S, Bauters C, et al. Prognostic importance of tissue Doppler-derived diastolic function in patients presenting with acute coronary syndrome: a bedside echocardiographic study. Eur J Echocardiogr 2008;9:594-8.
25. Dokainish H, Rajaram M, Prabhakaran D, et al. Incremental value of left ventricular systolic and diastolic function to determine outcome in patients with acute ST-segment elevation myocardial infarction: the echocardiographic substudy of the OASIS-6 trial. Echocardiography 2014;31:569-78.

26. Lin AK, Sippel C, Guppy-Coles J, et al. Abstract 11330: E/e' is a Powerful Predictor of Survival After a First-Ever Non-ST Elevation Myocardial Infarction. Circulation 2016;134(Suppl 1).A11330

27. Independent Hospital Pricing Authority. National efficient price determination 2016-17. Sydney, 2016.

28. Department of Health. Medicare benefits schedule book: Commonwealth of Australia, 2015.

29. Goldstein JA, Chinnaiyan KM, Abidov A, et al. The CT-STAT (Coronary Computed Tomographic Angiography for Systematic Triage of Acute Chest Pain Patients to Treatment) trial. J Am Coll Cardiol 2011;58:1414-22.

30. Hoffmann U, Truong QA, Schoenfeld DA, et al. Coronary CT angiography versus standard evaluation in acute chest pain. $N$ Engl $J$ Med 2012;367:299-308.

31. Brügger-Andersen T, Pönitz V, Staines $\mathrm{H}$, et al. B-type natriuretic peptide is a long-term predictor of all-cause mortality, whereas highsensitive C-reactive protein predicts recurrent short-term troponin T positive cardiac events in chest pain patients: a prognostic study. BMC Cardiovasc Disord 2008;8:34.

32. Lin A, Kwan C, Sippel J, et al. Abstract 11330: E/e' is a Powerful Predictor of Survival After a First-Ever Non-ST Elevation Myocardial Infarction. Circulation 2016;134:A11330.

33. Queensland Health. Wage rates - Health practitioners. $2017 \mathrm{https}: / /$ www.health.qld.gov.au/hrpolicies/wage_rates/health-practitioners (Accessed 15 Sept 2017).

34. Department of Community Safety. The State of Queensland (Department of Community Safety) annual report 2012-2013. Brisbane: Department of Community Safety, the State of Queensland, 2013. 